\title{
A practical training program for fluid milk defect judging should focus on initial training of panelists
}

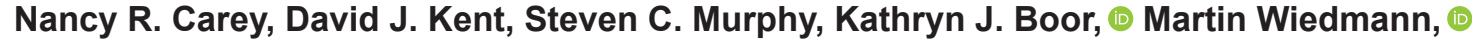 \\ and Nicole H. Martin* (B) \\ Milk Quality Improvement Program, Department of Food Science, Cornell University, Ithaca, NY 14853
}

\section{ABSTRACT}

The sensory quality of fluid milk is of great importance to processors and consumers. Defects in the expected odor, flavor, or body of the product can affect consumer attitudes toward the product and, ultimately, willingness to purchase the product. Although many methods of sensory evaluation have been developed, defect judging is one particular method that has been used for decades in the dairy industry for evaluating fluid milk. Defect judging is a technique whereby panelists are trained to recognize and rate a standard set of fluid milk defects that originate from various sources (e.g., microbial spoilage). This technique is primarily used in processing facilities where identification of sensory defects can alert personnel to potential quality control issues in raw material quality, processing, or good manufacturing practices. In 2014-2016, a preliminary study of defective milk judging screening and training was conducted by the Milk Quality Improvement Program at Cornell University (Ithaca, NY). The study, which included 37 staff and students from the Cornell community, used prescreenings for common odors and basic tastes, followed by uniform training to select, initially train, and retrain defect judges of unflavored high temperature, short time fluid milk. Significant improvements were seen in correct identification of defect attributes following initial training for all defect attributes, with the exception of fruity/fermented. However, following retraining, significant improvements were observed in only 2 defect attributes: cooked and milk carton. These results demonstrate that initial training is important for panelists to correctly identify fluid milk defect attributes, but that subsequent retraining should be tailored toward specific attributes. This study provides a resource for dairy industry stakeholders to use to develop relevant and efficient training methods for fluid milk defect judging panels.

Received July 26, 2019.

Accepted February 19, 2020

*Corresponding author: nhw6@cornell.edu
Key words: milk quality, sensory training

\section{INTRODUCTION}

Fluid milk quality is an important issue for the dairy industry. Methods for evaluating quality include microbiological, chemical, and sensory testing. In the area of sensory evaluation, several distinct approaches have been used to assess fluid milk quality, including defect or quality judging (Carey et al., 2005; Martin et al., 2012), analytical sensory tools (e.g., discrimination tests; Schiano et al., 2017), and consumer acceptance testing (Schiano et al., 2017). Fluid milk defect judging is a fast and practical method for dairy processing plant personnel to make rapid decisions on product quality. Trained personnel can quickly identify gross product quality defects and their potential sources and take steps to correct the problem or prevent reoccurrence (Drake, 2007). Other individuals in positions to assist the dairy industry can also, using the same terminology for consistency of terms, be trained to evaluate fluid milk defects in retail samples over shelf life (Carey et al., 2005; Martin et al., 2012). The USDA grading and American Dairy Science Association (ADSA) scorecard judging was developed in the early 1900s to grade finished products for quality and consistency and train students (Bodyfelt et al., 1988). The ADSA-based scoring provides an organized list of criticisms or defects that are frequently encountered. It facilitates the evaluation of many samples per session and aids efficiency and generation of numbers depicting general quality, along with defect descriptors (Bodyfelt et al., 2008). Panelists determine the level (slight, definite, or pronounced) of each detected defect in a fluid milk sample, compared with their concept of an ideal product, and then classify the overall flavor quality of the sample on a scale of 0 to 10 , with 9 to 10 indicating excellent quality (Clark and Costello, 2009).

Defect judging does have some limitations. Results do not describe the entire sensory profile of the product, and the terms are not as specific as in traditional descriptive analysis, which makes identification and 
scoring more subjective when defect judging is used. Also, point reductions are not consistent from defect to defect and are not linear within a specific defect, which may preclude the use of statistical analysis (Bodyfelt et al., 2008). Further, results may not be strictly associated with consumer acceptance or preference, because trained judges may expect a higher quality than consumers demand (i.e., trained judges might be more critical; Lawless and Claassen, 1993; Drake, 2007), or, because of their training, may be more sensitive to certain attributes (Chapman et al., 2002). These factors make defect judging unsuitable for use in product development or market research (Drake, 2007). Despite any drawbacks, traditional dairy processing education, which includes defect judging, has contributed to the attainment of the high sensory quality products that consumers enjoy today and will continue to affect the dairy industry alongside more advanced sensory tools used in new product development (Bodyfelt et al., 2008). The goal of this study was to evaluate the effects of a standard defect judging training protocol on the ability of panelists to correctly identify defect attributes after initial training and retraining.

\section{MATERIALS AND METHODS}

\section{Panelist Recruitment, Attendance, and Attrition}

Undergraduate and graduate students and staff (n $=37$ ) from Cornell University were recruited (based on willingness to participate, availability, milk liking, and no reported allergies or taste problems) for training to evaluate HTST fluid white milk over shelf life from 2014 to 2016. Recruits consisted of 11 men and 26 women between the ages of 18 and $63 \mathrm{yr}$. The research protocol was approved by the Cornell University Institutional Review Board that protects human subjects, and informed consent was obtained from volunteers before training. At the end of training, recruits were paid for time spent training for the defect judging panel.

\section{Panelist Prescreening}

To ensure that qualified individuals were recruited for the remainder of the study to demonstrate study objectives, recruits' sensory abilities were prescreened, beginning with a modified version of the International Organization for Standardization's recommendations for odor recognition (ISO, 2009). Odor recognition samples included sweetened condensed milk, buttermilk, grape nuts-type cereal in milk, chopped wet alfalfa hay, chopped mushrooms, provolone cheese, pineapple juice, orange juice, lemon juice, household bleach, vinegar, and ammonia. Basic taste samples (sweet, sour, salty, and bitter) were prepared at 3 levels of intensity. An overall score of at least $65 \%$ (38 out of 58 points) for the combination of odor recognition and identification and ranking of basic tastes was required to continue to initial training.

\section{Preparation of Defective Milk Samples}

Briefly, for all samples, half gallons of $2 \%$ and skim pasteurized milk in plastic containers were used. All samples were made from milk that had the same code date (i.e., were manufactured in the same batch). Milk for most of the training sessions was obtained through the Cornell Dairy supply chain within $48 \mathrm{~h}$ of pasteurization. If purchased from a retail store, the freshest milk (with the longest code date) was requested from the back cooler rather than choosing milk from a lighted display case, to avoid light oxidation defects (Chapman et al., 2002). Containers were protected from light and kept cold $\left[<4.0^{\circ} \mathrm{C}\left(39.2^{\circ} \mathrm{F}\right)\right.$ without freezing $]$ after purchase. A portion, removed during sample preparation, was tasted by the panel leader to ensure freshness and the absence of light oxidized, strongly cooked, or any other flavor defect.

Perishables (e.g., buttermilk, cheese) were purchased just before each preparation and stored at $<4.0^{\circ} \mathrm{C}$ $\left(39.2^{\circ} \mathrm{F}\right)$ without freezing. Stored nonperishable products (e.g., vinegar) were held at the appropriate temperature. All ingredients were transferred as aseptically as possible, using a clean apparatus for transfer and containment for each preparation. Containers were gently totally inverted 25 times for thorough mixing (Duncan et al., 2004) (1) before removal of milk from any retail containers, (2) after addition of ingredients, (3) after transfer of portions back to the original container, and (4) before pouring into individual cups for tasting. Approximately $1 \mathrm{oz}$. of each sample was poured into a 5-oz. odor-free plastic cup (Fabrical Corp., Kalamazoo, MI). Lids were applied after each flavor was poured to prevent possible carryover of odors to other cups. For the tasting portion of the lecture-based training and for reference samples, cups were numbered with single digits. For all other training, cups were randomly numbered with a triple-digit code to avoid bias (Lawless and Heymann, 1999). Additional information can be accessed at www.github.com/mqip/sensory-training.

\section{Pretest}

Before beginning initial training or retraining, a pretest was administered. The pretest consisted of a set of defective $2 \%$ milk samples $(n=10)$ and control 
$2 \%$ milk samples $(\mathrm{n}=8)$ in the morning and a set of defective skim milk samples $(\mathrm{n}=10)$ and control skim milk samples $(\mathrm{n}=8)$ in the afternoon of the same day (Figure 1). All samples were presented in a welllit, temperature-controlled room free of confounding odors and with privacy walls between panelist stations. Samples were warmed to $15.5^{\circ}$ to $21^{\circ} \mathrm{C}\left(60^{\circ}\right.$ to $\left.70^{\circ} \mathrm{F}\right)$ using a microwave, and then swirled to ensure even heat distribution. A set length of time for warming, determined by the number of samples per set, provided as much consistency as possible among samples of each panelist's set, as well as among all panelists' sets. Panelists evaluated the samples according to procedures described previously (Chapman et al., 1998). Sample tasting order was randomized both between sets and among participants. Recruits were asked to correctly identify each 3-digit blind-coded control milk or defect, rate its intensity, and score it according to the materials provided.

\section{Initial Training}

Initial training began with approximately $4 \mathrm{~h}$ of lectures and hands-on activities over d 1 to 4 (Figure 1), beginning with a 1.5-h lecture-based training on each of 2 consecutive days covering (1) sources of flavor, odor, and body defects; (2) proper tasting procedures; (3) milk defect descriptions; and (4) sample scoring. After the first lecture, recruits participated in exercises using fresh retail "control" milks, ranking them by viscosities (whole, $2 \%, 1 \%$, skim, and skim milk with $20 \%$ added water), cooked flavors (HTST vs. ultrapasteurized), and sweetness (2\% milk with $20 \%$ added water vs. $2 \%$ vs. $2 \%$ lactose-reduced). Following the second lecture, recruits were introduced to acid, feed, fruity/fermented, malty, and rancid defective milk odors, and were asked to match treated milks to their ingredients (i.e., buttermilk, alfalfa, pineapple juice, Grape-Nuts cereal in milk, and provolone cheese, respectively). Further, recruits were introduced to the terms foreign/chemical, lacks freshness, cowy, barny, and unclean. Basic sensory terms were presented, stressing the importance of odors and retronasal smell, which is often mistaken for "taste" (Lawless and Heymann, 1999; Shepherd, 2006). Guidelines for panelists, as well as proper tasting techniques, were also presented in this training and can be found at www.github.com/mqip/sensory-training. An unscored quiz was administered on d 3 and 4 of training (Figure 1). Quizzes comprised 3-digit blind-coded randomized defective milk samples $(\mathrm{n}=10)$ and control milks $(\mathrm{n}=2)$, as well as a set of defective milk reference samples (numbered 1 to 11 with a corresponding list of defects) for comparison on d 3, and the same set of quiz milks (re-randomized) without the reference set on d 4 (Figure 1). A fresh $2 \%$ control sample was used as a warm-up, followed by the 12 quiz samples. On both quiz days, samples were presented in a random order, but in the same order for all recruits, and answers were supplied at the end of each session, with instructions encouraging participants to retaste samples as needed.

On the d 5 through 10 of training (approximately 45 min each day), panelists were presented with defective $2 \%$ milk samples (d 5 through 7 ) and defective skim milk samples (d 8 through 10), as well as controls (i.e., corresponding milk samples with no defects; Figure 1). Milk defects for tasting were initially presented in 2 groups (i.e., subtle defects and stronger defects) so as not to confound the trainees' senses. Subtle defects, flat, milk carton, cooked, feed, and light oxidized, were presented first, followed by the stronger and more serious (i.e., most often microbial) defects malty, acid, rancid, fruity/fermented, and bitter. Milk off-flavors (based on definitions by ADSA, 2005), their categories, causes, prevention, scoring, and sample preparation were thoroughly described as the samples were tasted. During training, recruits attended practice test sessions on 2 sets of 3 consecutive days, with $2 \%$ samples the first week, and skim samples the second week (d 5 to 7 and d 8 to 10, respectively; Figure 1).

\section{Posttest}

Initial training and retraining were followed by a posttest (the same samples as in the pretests but re-randomized). Samples were prepared following the same sample preparation procedures and presented under the same environmental conditions described for the pretest. Panelists who received $\geq 70 \%$ on the posttest were eligible to join or remain on the defect panel. Panelists receiving $<70 \%$ failed the posttest and were given the option to retry the initial training and posttest during the next scheduled panel training. However, panelists were permanently disqualified from serving on the defect panel if they received $<70 \%$ on both the initial training and the retraining posttests.

\section{Retraining}

Panelists were required to attend retraining every 6 mo after their initial training to remain on the defect judging panel. Retraining followed the same progression and methods as the pretest, initial training, and posttest, except that the lecture-based training was excluded from retraining. As with the initial training, 

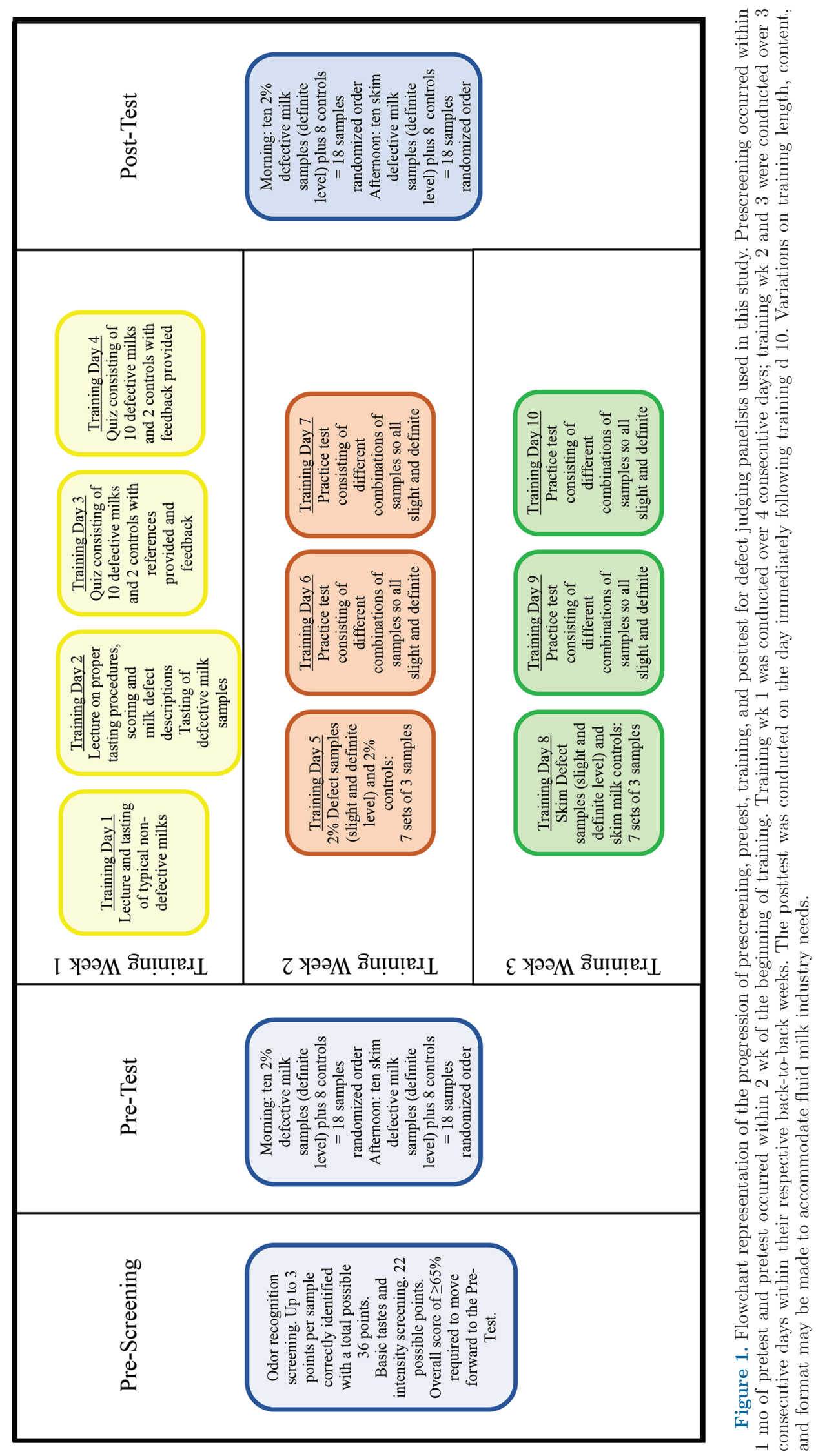
panelists who received $\geq 70 \%$ on the posttest remained on the defect panel. Panelists receiving $<70 \%$ failed the posttest and were given the option to retry the retraining and posttest during the next scheduled panel training. To improve efficiency of the overall training program, and to account for panelists joining the study at different times through the study period, training for new recruits (initial training) and existing panelists (retraining) was conducted during the same sessions. A total of 6 sessions were conducted during the study period.

\section{Data Capture and Analysis}

All basic tastes and defective milk judging data were captured and summarized throughout the study using the Compusense Cloud sensory evaluation software (Compusense Inc., Guelph, ON, Canada). All statistical analyses were conducted in R (R Core Team, 2016). A logistic linear model was performed using the glm function in $\mathrm{R}$ to compare pretest and posttest results for both initial training and retraining. Each observation consisted of one prepared sample and was regarded as a "success" if the prepared defect was marked appropriately by the panelist, and a "failure" otherwise. A 3-way interaction of "pre/post" (whether the test was occurring before or after a training session), "initial" (whether this test was associated with the panelist's first training session), and "defect" (what the prepared defect was), and associated 2-way interactions and main effects were used. Milk type (skim or $2 \%$ ) was included as a blocking effect. Contrasts between pre/post and initial/retraining were assessed using the lsmeans package (Lenth, 2016) in R. Data and statistical analysis code can be found at www.github.com/mqip/sensory -training.

\section{RESULTS}

\section{Panelist Prescreening}

An initial prescreening of new panelists $(\mathrm{n}=31$, not including 6 preexisting panelists) for their ability to identify basic tastes and odors was conducted to screen out any panelists with an impairment of the sense of taste or smell, and for ranking of various basic taste intensities to screen for the loss of taste sensitivity (Lawless and Heymann, 1999). Of the 31 panelists prescreened, 23 passed (1 panelist who passed subsequently dropped out of the study), 2 failed and upon subsequent prescreening passed, and 6 failed and did not attempt to retake the prescreening. This resulted in 24 panelists who passed the basic taste and odor prescreening and remained in the study.

\section{Initial Training}

Initial training was conducted for the 30 panelists remaining in the study following the prescreening (24 panelists who passed prescreening and 6 pre-existing experienced panelists who were exempted from screening). Panelists were exempted from screening if they were already members of the panel before the start of this study; however, pre-existing panelists participated in the initial and retraining as well as pre- and posttests for both trainings. Significant improvements were seen between pre- and posttest scores for the control and every defect tested (i.e., acid, bitter, cooked, feed, flat, light oxidized, malty, milk carton, and rancid) with the exception of fruity/fermented, which still showed a trend toward improvement $(P=0.08$; Table 1 , Figure 2). Odds ratios ranged from a low of 1.5 (95\% CI: 1.2-2.0) for control samples, for which $48 \%$ of samples were identified correctly before training and $59 \%$ were

Table 1. Summary of fluid milk defect judging scores before and after initial training by defect attribute

\begin{tabular}{llccc}
\hline & & \multicolumn{2}{c}{ Percent correct } & \\
\cline { 3 - 4 } Attribute & $\begin{array}{l}\text { Samples } \\
\text { evaluated }(\mathrm{n})\end{array}$ & Pretest & Posttest & $\begin{array}{c}\text { Odds ratio } \\
(95 \% \text { CI })\end{array}$ \\
\hline Acid & 60 & 68 & 93 & $6.5(2.1-20.5)$ \\
Bitter & 60 & 67 & 98 & $29.5(3.8-228.6)$ \\
Control & 480 & 48 & 59 & $1.5(1.2-2.0)$ \\
Cooked & 60 & 23 & 87 & $21.4(8.2-55.5)$ \\
Feed & 60 & 55 & 98 & $48.3(6.3-371.4)$ \\
Flat & 60 & 37 & 82 & $7.7(3.3-17.8)$ \\
Fruity/fermented & 60 & 85 & 95 & $3.4(0.9-13.1)$ \\
Light oxidized & 60 & 27 & 72 & $7.0(3.1-15.5)$ \\
Malty & 60 & 60 & 97 & $19.3(4.3-86.8)$ \\
Milk carton & 60 & 22 & 60 & $5.4(2.4-12.1)$ \\
Rancid & 60 & 50 & 85 & $5.7(2.4-13.5)$ \\
\hline
\end{tabular}


Pre- or post-training

- Pre

- Post

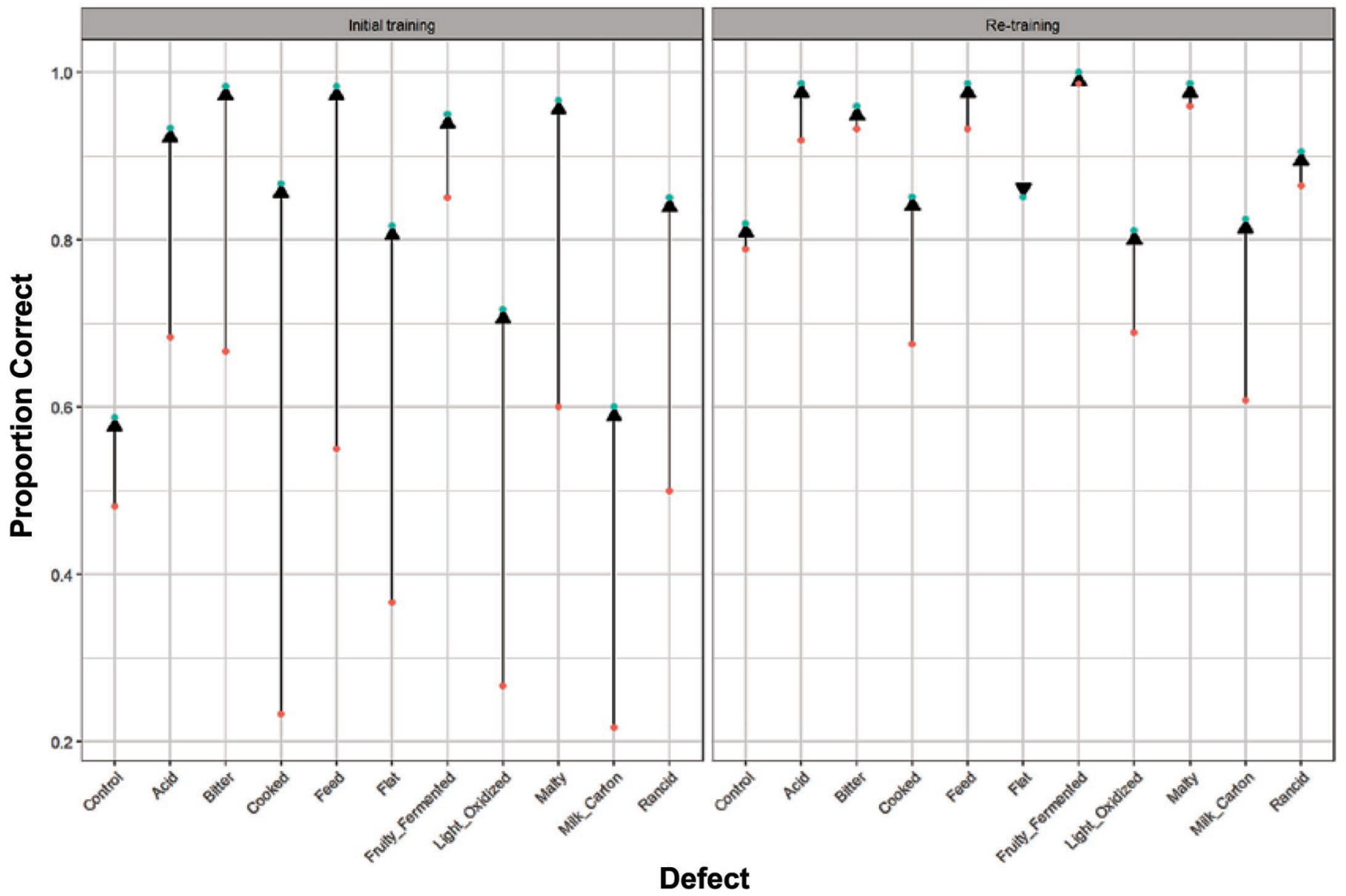

Figure 2. Proportion of defective milk samples correctly identified by panelists during pretest and posttest evaluation for initial training and retraining. Direction of arrow indicates increase in proportion correct $(\uparrow)$ or decrease in proportion correct $(\downarrow)$.

correctly identified after training, to 48.3 (95\% CI: 6.3-371.4) for feed defect, where $55 \%$ of samples were correctly identified before training and $98 \%$ of samples were correctly identified after training (Table 1, Figure $2)$.

\section{Panelist Retraining}

Panelists were evaluated for panel participation based on performance following initial training. Of the original 30 panelists participating in initial training, 8 failed the initial training posttest (i.e., received $<70 \%$ on initial training posttest) and declined to retry the initial training, 2 retried the initial training and subsequently passed, and 1 retried the initial training and subsequently failed, resulting in disqualification from the panel (Table 2). Thus, a total of 21 panelists were eligible for retraining (1-4 sessions per panelist during the study period based on initial training date). Of those 21 panelists, 9 successfully passed either the initial training or one or more retraining but ultimately did not return to the panel, 2 failed a previous retraining and did not attempt to retry the retraining, and 10 were retained on the panel throughout the remainder of the study (Table 2).

Overall, pooled retraining scores show trends for defect detection improvement after retraining for certain attributes, but improvement was found to be significant only for cooked and milk carton defects, with odds ratios of 2.8 (95\% CI: $1.23-6.25)$ and 3.0 (95\% CI: 1.4-6.5), respectively (Table 3, Figure 2). Several attributes showed little or no improvement in percent identified correctly after retraining, including bitter, flat, fruity/fermented, and malty (Table 3, Figure 2). 
Table 2. Summary of training outcomes for initial training and retraining of 30 study participants in the initial training

\begin{tabular}{|c|c|c|c|c|c|c|}
\hline \multirow[b]{2}{*}{ Training session ${ }^{1}$} & \multirow[b]{2}{*}{$\begin{array}{c}\text { Passed } \\
\text { (no.) }\end{array}$} & \multirow[b]{2}{*}{$\begin{array}{c}\text { Failed } \\
\text { (no.) }\end{array}$} & \multicolumn{4}{|c|}{ Session not taken ${ }^{2}$} \\
\hline & & & $\begin{array}{l}\text { Passed previous training } \\
\text { and did not return (no.) }\end{array}$ & $\begin{array}{l}\text { Failed previous training and } \\
\text { did not attempt retry (no.) }\end{array}$ & $\begin{array}{l}\text { Disqualified }{ }^{3} \\
\text { (no.) }\end{array}$ & $\begin{array}{l}\text { Training not } \\
\text { needed }^{4} \text { (no.) }\end{array}$ \\
\hline First retraining & 15 & 1 & 4 & 8 & 1 & 1 \\
\hline Second retraining & 9 & 1 & 5 & 9 & 1 & 5 \\
\hline Third retraining & 6 & 0 & 7 & 10 & 1 & 6 \\
\hline
\end{tabular}

${ }^{1}$ For each training session the sum of the number passed, number failed, and number of participants that did not take the session is equal to 30 .

${ }^{2}$ For each training session several participants did not participate in that particular session.

${ }^{3}$ Participants were disqualified after 2 posttest failures (e.g., receiving $<70 \%$ on initial or retraining posttest).

${ }^{4}$ Participants were required to undergo retraining every 6 mo. Based on the date the participant took the initial training, they may not have been eligible for retraining at the time of every retraining session.

${ }^{5}$ Of the 21 panelists who passed the initial training, 3 failed their first try and passed upon retry.

${ }^{6} \mathrm{NA}=$ not applicable.

\section{Correct Identification of Defect Attributes}

Defect attributes incorrectly assigned by panelists were compared with the actual sample defect attributes both before and after initial training and retraining to identify differences in training efficacy by defect attribute (Figure 3; Supplemental Figure S1; https:/ /doi.org/10.3168/jds.2019-17336). Retention of ability to detect defect attributes generally followed 1 of the following 3 trends: (1) defect attribute correctly identified following training, (2) defect attribute incorrectly marked as another defect following training, and (3) defect attribute incorrectly marked as control following training. The defect attributes that were most frequently correctly identified following initial training and retraining were acid, bitter, feed, fruity/fermented, and malty. For example, before initial training, panel- ists misidentified a total of 27 feed samples, but following initial training, they misidentified only 1 feed defect sample (Figure 3a). Further, before retraining, panelists misidentified 5 feed defect samples by, whereas following retraining, they misidentified only 1 feed defect sample as malty (Figure 3a).

Defect attributes that were frequently incorrectly marked as another defect following training included light oxidized, milk carton, and rancid. For example, panelists misidentified 47 milk carton defect samples before initial training, with the most commonly misidentified defect being light oxidized $(\mathrm{n}=10$; Figure 3b). Following initial training, only 24 milk carton defect samples were misidentified, with the most commonly misidentified defect remaining light oxidized (n $=11$; Figure 3b). Similarly, before retraining, 29 milk carton defect samples were misidentified, with the most

Table 3. Summary of fluid milk defect judging scores before and after retraining by defect attribute

\begin{tabular}{|c|c|c|c|c|c|}
\hline \multirow[b]{2}{*}{ Attribute } & \multirow{2}{*}{$\begin{array}{l}\text { Samples } \\
\text { evaluated }^{1}(\mathrm{n})\end{array}$} & \multicolumn{2}{|c|}{ Percent correct } & \multirow{2}{*}{$\begin{array}{c}\text { Odds ratio }^{2} \\
(95 \% \text { CI })\end{array}$} & \multirow[b]{2}{*}{$P$-value } \\
\hline & & Pretest & Posttest & & \\
\hline Acid & 74 & 92 & 99 & $6.4(0.8-54.8)$ & 0.088 \\
\hline Bitter & 74 & 93 & 96 & $1.7(0.4-7.4)$ & 0.471 \\
\hline Control & 592 & 79 & 82 & $1.2(0.9-1.6)$ & 0.188 \\
\hline Cooked & 74 & 68 & 85 & $2.8(1.2-6.2)$ & 0.013 \\
\hline Feed & 74 & 93 & 99 & $5.3(0.6-46.4)$ & 0.132 \\
\hline Flat & 74 & 85 & 85 & $1.0(0.4-2.5)$ & 1.000 \\
\hline Fruity/fermented & 74 & 99 & 100 & $\mathrm{NA}^{3}$ & NA \\
\hline Light oxidized & 74 & 69 & 81 & $1.9(0.9-4.1)$ & 0.090 \\
\hline Malty & 74 & 96 & 99 & $3.1(0.3-30.3)$ & 0.334 \\
\hline Milk carton & 74 & 61 & 82 & $3.0(1.4-6.5)$ & 0.004 \\
\hline Rancid & 74 & 86 & 91 & $1.5(0.5-4.2)$ & 0.441 \\
\hline
\end{tabular}

${ }^{1}$ Panelists who passed initial training and went on to retraining $(\mathrm{n}=30)$ evaluated a set of 1 of each defective milk sample and 8 control samples during the pretest and posttest. Panelists participated in a varying number of retraining sessions based on the date they completed the initial training.

${ }^{2}$ Odds ratios were calculated from contrasts between pre- and post-initial training scores.

${ }^{3} \mathrm{NA}=$ not applicable. 

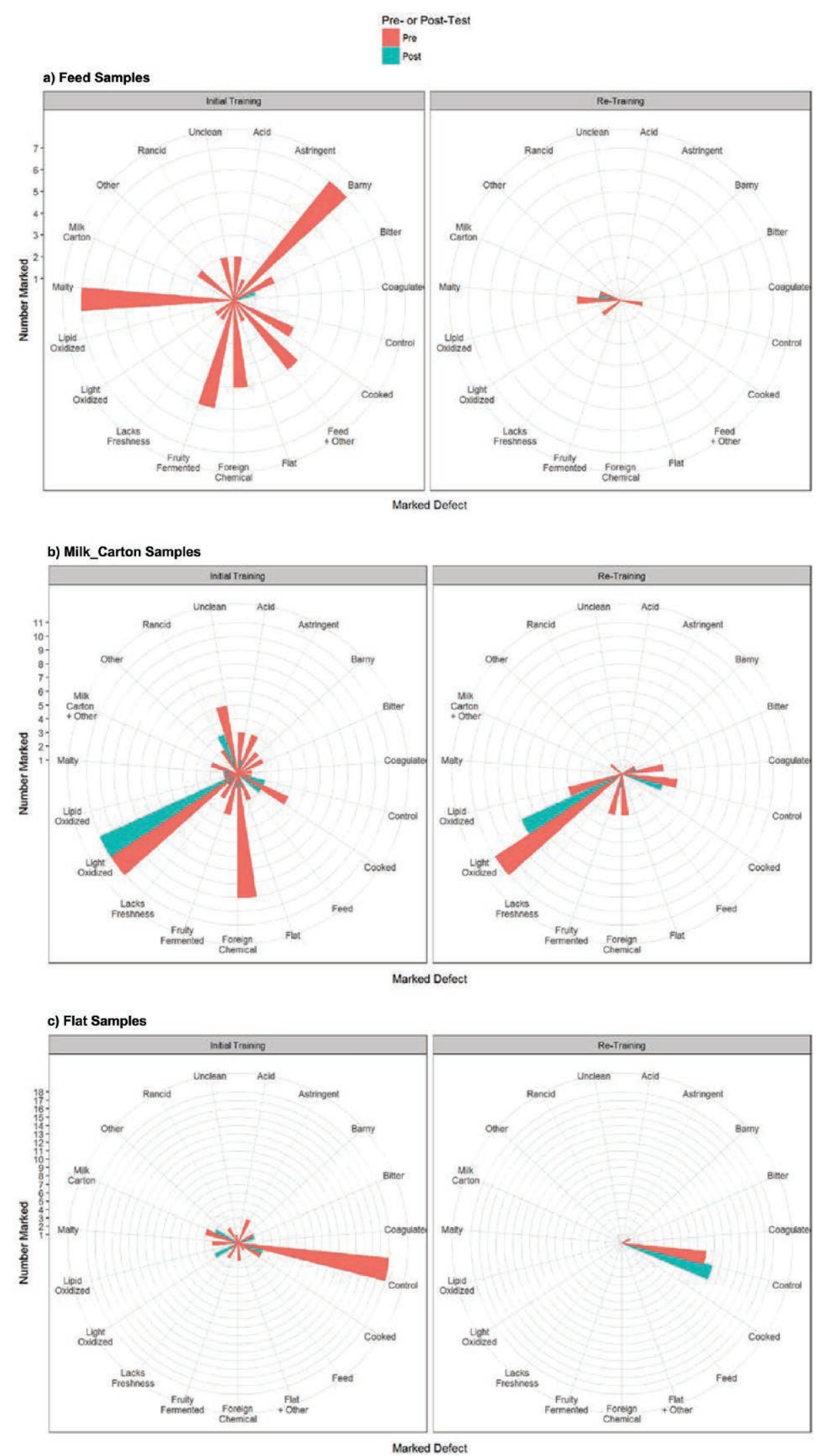

Figure 3. Radial plots of incorrectly identified (a) feed, (b) milk carton, and (c) flat defective milk samples during pretest and posttest evaluation for initial training and retraining of panelists. A single defective milk sample may be marked as more than one incorrect defect. 
commonly misidentified defect being light oxidized (n $=11$ ). Following retraining, only 13 milk carton defect samples were misidentified, again with light oxidized $(\mathrm{n}=8)$ being the most commonly misidentified defect (Figure 3b).

Finally, flat and cooked defect attributes were frequently misidentified as control following training. Before initial training, 38 flat defect samples were misidentified, with the most commonly misidentified defect being control $(\mathrm{n}=18$; Figure $3 \mathrm{c})$. Following initial training, 11 samples were misidentified as control $(\mathrm{n}=3)$, light oxidized $(\mathrm{n}=3)$, milk carton $(\mathrm{n}=3)$, or bitter $(\mathrm{n}=2)$. Before retraining, 11 flat defect samples were misidentified as control $(\mathrm{n}=10)$ or bitter $(\mathrm{n}=$ 1), whereas following retraining, 11 flat defect samples were misidentified as control (Figure 3c).

\section{DISCUSSION}

\section{Effective Training Programs}

Our study showed that panelists' abilities to correctly identify fluid milk defects significantly improved for all defects, with the exception of fruity/fermented, upon initial training. Further, misidentified defects before and after initial training show that for many defects (e.g., acid, bitter, feed, and malty), there were a large number of initial misidentifications before training; however, once training occurred that number dropped dramatically, indicating that the panelist was able to retain that knowledge and correctly identify these defects. Previous studies have been conducted to determine the effects of training on sensory evaluation of various foods. Those studies have found that although panelists generally benefited from increased hours of training, relatively few hours of training had a significant effect on panelists' abilities to correctly identify product attributes, especially if there was little variability in the product characteristics (Wolters and Allchurch, 1994; Chambers et al., 2004). Indeed, in our study, panelists participated in approximately $7.5 \mathrm{~h}$ of initial training, which significantly improved their ability to detect most fluid milk defect attributes, with the exception of fruity/fermented, which was identified correctly by more than $80 \%$ of the panelists before training. There is no rule for what determines optimal individual panelist or collective panel performance (Drake, 2007). The length of training time needed has been proposed to be determined by the complexity of the product or number of attributes that will be profiled, as well as the panel's ability to replicate results (Drake, 2004). If only a few distinct attributes are being profiled, a few hours of training could suffice; however, if many attributes are profiled, several hundred hours and a significant financial commitment may be required to ensure panelists operate in unison as a calibrated instrument that can replicate results (Lawless and Heymann, 1999, Drake, 2007). This approach tends to be too expensive for day-to-day quality assurance (Lawless and Heymann, 1999). Our results show that a training program of approximately $7.5 \mathrm{~h}$ provides adequate training for fluid milk defect judging, meaning that panelists significantly improved in their ability to accurately detect fluid milk defect attributes.

The goal of fluid milk defect judging is to provide rapid, practical, and cost-effective feedback regarding the quality and consistency of fluid milk products, particularly at the processing plant level, as it requires a relatively small number of trained panelists (3 to 6 people) for product evaluation. As with any tool used to assess the quality of fluid milk, the accuracy and precision of a defect judging panel is critical to its overall effectiveness and the quality of the feedback provided. Therefore, developing a training program that is robust and focuses on panel outcomes (i.e., correct identification of product defects) is of great interest to the dairy industry. Importantly, results from our study suggest that retraining of fluid milk defect panelists should focus on the subset of defect attributes that showed significant improvement in percentage correct after retraining (i.e., cooked and milk carton). Prioritizing training efforts will allow organizations using fluid milk defect judging to maintain a well-trained and functional defect judging panel and minimize training time and use of resources.

\section{Misidentified Fluid Milk Defects}

Our study indicated that certain fluid milk defect attributes, including light oxidized and milk carton, were more commonly misidentified (as other flavor attributes) than others, even after retraining. Further, we identified defect attributes that were commonly misidentified as control even after retraining, including cooked and flat. Although these defect attributes are generally considered subtle, misidentification at the processing level may have implications for consumer acceptance. For example, studies have shown that consumer acceptance of fluid milk with light oxidation defects due to exposure to fluorescent (Potts et al., 2017) or LED (light-emitting diode; Martin et al., 2016) light sources is significantly reduced compared with fluid milk that has not been exposed to light. Our findings suggest that detection of light oxidized defects may be reduced, even by trained defect judging panelists. These studies highlight the conclusion from our study that 
retraining should focus on a subset of defect attributes, including light oxidized, to optimize the defect judging panelists' ability to correctly identify these attributes and ultimately resolve the source of the defect.

\section{Blueprint for a Defect Judging Training Program}

The use of in-house sensory analysis, or "flavoring," is commonly used in the dairy industry on samples retained throughout shelf life. This end-of-shelf-life analysis allows processors to evaluate their product quality, as well as to inform their code date. Defect judging specifically provides a rapid, cost-effective tool for dairy plants and a standardized vocabulary with which to identify quality problems and score their potential effect on product acceptance (Lawless and Claassen, 1993). Further, as fluid milk processors strive to obtain food quality certifications, they may be required to implement a sensory evaluation program. For example, the internationally recognized Safe Quality Foods quality code requires that a sensory evaluation program "shall be in place to ensure alignment with agreed customer requirements" (SFQI, 2017). Although the importance of these analyses to a fluid milk processor cannot be understated, it is generally unknown across the industry what training procedures are used for personnel who perform fluid milk defect judging. Personal communications with industry professionals indicate a lack of standardized training methods and evaluation criteria for training methods. This study provides a detailed approach to the selection and training of a fluid milk defect judging panel that can be used by the dairy industry to implement standardized protocols for in-house use. Importantly, detailed protocols and training materials are made available in this article and on www.github.com/mqip/sensory-training, facilitating industry implementation of the type of training reported here.

\section{CONCLUSIONS}

Providing the dairy industry with standardized tools to evaluate fluid milk quality is critical to achieve the goals of the fluid milk industry, including improving product quality, extending shelf life, or implementing overall quality programs such as the Safe Quality Foods quality code. One important tool to achieve this goal is sensory defect judging, which is a rapid and low-input method for evaluating fluid milk for sensory defects. Our study shows that brief initial panelist training, which can be applied by fluid milk processing personnel, significantly affects panel outcomes, but that retraining should target select defect attributes.

\section{ACKNOWLEDGMENTS}

This study was supported by the New York State Dairy Promotion Advisory Board, dairy producers committed to funding research to advance the New York State dairy industry, through the New York State Department of Agriculture and Markets. The authors thank the staff and students of the Milk Quality Improvement Program (Cornell University, Ithaca, NY) and the defect judging panelists for assistance with this study. The authors have not stated any conflicts of interest.

\section{REFERENCES}

ADSA. 2005. Committee on Evaluation of Dairy Products. Score Card Sub-Committee. American Dairy Science Association (ADSA), Champaign, IL.

Bodyfelt, F. W., M. A. Drake, and S. A. Rankin. 2008. Developments in dairy foods sensory science and education: From student contests to impact on product quality. Int. Dairy J. 18:729-734. https: //doi.org/10.1016/j.idairyj.2008.03.011.

Bodyfelt, F. W., J. Tobias, and G. M. Trout. 1988. The Sensory Evaluation of Dairy Products. Van Nostrand Reinhold, New York, NY.

Butler, A. C., and H. L. J. M. Roediger. 2008. Feedback enhances the positive effects and reduces the negative effects of multiple-choice testing. Memory Cognition 36:604-616.

Carey, N. R., S. C. Murphy, R. Zadoks, and K. J. Boor. 2005. Shelf lives of pasteurized fluid milk products in New York State: A ten year study. Food Prot. Trends 25:102-113.

Chambers, D. H., A.-M. A. Allison, and E. Chambers IV.. 2004. Training effects on performance of descriptive panelists. J. Sens. Stud. 19:486-499. https://doi.org/10.1111/j.1745-459X.2004.082402.x.

Chapman, K., L. Rosenberry, D. Bandler, and K. Boor. 1998. Lightoxidized flavor development and vitamin A degradation in chocolate milk. J. Food Sci. 63:930-934. https://doi.org/10.1111/j.1365 -2621.1998.tb17929.x.

Chapman, K., L. Whited, and K. Boor. 2002. Sensory threshold of light-oxidized flavor defects in milk. J. Food Sci. 67:2770-2773. https://doi.org/10.1111/j.1365-2621.2002.tb08813.x.

Clark, S., and M. Costello. 2009. Dairy products evaluation competitions. Pages 43-71 in The Sensory Evaluation of Dairy Products. S. Clark, M. Costello, M. Drake, and F. Bodyfelt, ed. Springer US, New York, NY.

Drake, M. 2004. ADSA Foundation Scholar Award: Defining dairy flavors. J. Dairy Sci. 87:777-784. https://doi.org/10.3168/jds.S0022 -0302(04)73221-X.

Drake, M. A. 2007. Invited review: Sensory analysis of dairy foods. J. Dairy Sci. 90:4925-4937. https://doi.org/10.3168/jds.2007-0332.

Duncan, S. E., B. R. Yaun, and S. S. Sumner. 2004. Chapter 09: Microbiological methods for dairy products. Pages 249-268 in Standard Methods for the Examination of Dairy Products. H. M. Wehr and J. F. Frank, ed. American Public Health Association, Washington, DC.

ISO. 2009. Milk and milk products-Sensory analysis-Part 1: General guidance for the recruitment, selection, training and monitoring of assessors. ISO 22935-1:2009 (IDF 99-1:2009). International Organization for Standardization (ISO), Geneva, Switzerland.

Lawless, H. T., and M. R. Claassen. 1993. Validity of descriptive and defect-oriented terminology systems for sensory analysis of fluid milk. J. Food Sci. 58:108-112. https://doi.org/10.1111/j.1365-2621 .1993.tb03221.x.

Lawless, H. T., and H. Heymann. 1999. Principles of good practice. Pages 83-115 in Sensory Evaluation of Food: Principles and Practices. Springer US, Boston, MA. 
Lenth, R. V. 2016. Least squares means: The R package lsmeans. J. Stat. Softw. 69:1-33. https://doi.org/10.18637/jss.v069.i01.

Martin, N., N. Carey, S. Murphy, D. Kent, J. Bang, T. Stubbs, M. Wiedmann, and R. Dando. 2016. Exposure of fluid milk to LED light negatively affects consumer perception and alters underlying sensory properties. J. Dairy Sci. 99:4309-4324. https://doi.org/10 $.3168 /$ jds.2015-9603.

Martin, N. H., N. R. Carey, S. C. Murphy, M. Wiedmann, and K. J. Boor. 2012. A decade of improvement: New York State fluid milk quality. J. Dairy Sci. 95:7384-7390. https://doi.org/10.3168/jds 2012-5767.

Potts, H. L., K. N. Amin, and S. E. Duncan. 2017. Retail lighting and packaging influence consumer acceptance of fluid milk. J. Dairy Sci. 100:146-156. https://doi.org/10.3168/jds.2016-11673.

Schiano, A. N., W. S. Harwood, and M. A. Drake. 2017. A 100-Year Review: Sensory analysis of milk. J. Dairy Sci. 100:9966-9986. https://doi.org/10.3168/jds.2017-13031.

SQFI. 2017. SQF Quality Code Edition 8. SQF Institute (SQFI), Arlington, VA. Accessed Apr. 10, 2020. https://www.sqfi.com/wp -content/uploads/2019/07/SQF-Quality-Code-ed-8.1-FINAL.pdf.

Shepherd, G. M. 2006. Smell images and the flavour system in the human brain. Nature 444:316-321. https://doi.org/10.1038/ nature 05405 .
Small, R. V. 1996. Dimensions of interest and boredom in instructional situations. Page 16 in Proceedings of Selected Research and Development Presentations at the 1996 National Convention of the Association for Educational Communications and Technology, Indianapolis, IN.

R Core Team. 2016. R: A Language and Environment for Statistical Computing. R Foundation for Statistical Computing, Vienna, Austria.

Wolters, C. J., and E. M. Allchurch. 1994. Effect of training procedure on the performance of descriptive panels. Food Qual. Prefer. 5:203-214. https://doi.org/10.1016/0950-3293(94)90036-1.

\section{ORCIDS}

Kathryn J. Boor ๑ https://orcid.org/0000-0001-6810-3434

Martin Wiedmann (1) https://orcid.org/0000-0002-4168-5662

Nicole H. Martin (® https://orcid.org/0000-0003-1704-0634 